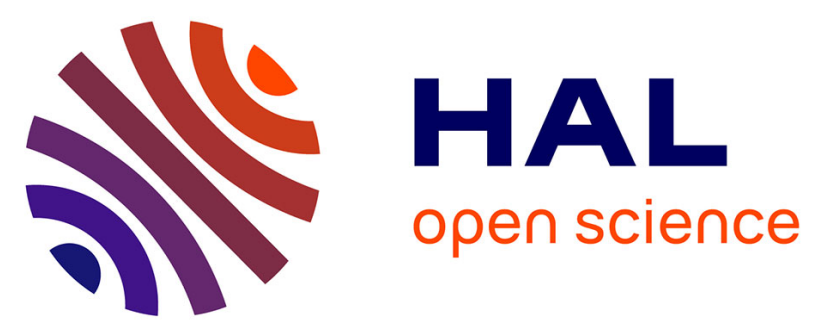

\title{
French Therapeutic Education Programme Aimed at Improving the Quality of Life of Laryngectomised Patients and their Close Relations: the Three Stages (Observational and Interventional Randomised) of the Study "PETAL"
}

Natacha Heutte, Moumainn Abouzayd, Laetitia Plisson, Mélanie Trocmé, Mélanie Seillier, Véronique Christophe, Lydia Guittet, Guillaume Grandazzi, Emmanuel Babin

\section{To cite this version:}

Natacha Heutte, Moumainn Abouzayd, Laetitia Plisson, Mélanie Trocmé, Mélanie Seillier, et al.. French Therapeutic Education Programme Aimed at Improving the Quality of Life of Laryngectomised Patients and their Close Relations: the Three Stages (Observational and Interventional Randomised) of the Study "PETAL". Journal of Cancer Education, 2018, 34 (4), pp.823-830. 10.1007/s13187-0181380-2 . hal-02149944

\section{HAL Id: hal-02149944}

https://hal-normandie-univ.archives-ouvertes.fr/hal-02149944

Submitted on 6 Jun 2019

HAL is a multi-disciplinary open access archive for the deposit and dissemination of scientific research documents, whether they are published or not. The documents may come from teaching and research institutions in France or abroad, or from public or private research centers.
L'archive ouverte pluridisciplinaire HAL, est destinée au dépôt et à la diffusion de documents scientifiques de niveau recherche, publiés ou non, émanant des établissements d'enseignement et de recherche français ou étrangers, des laboratoires publics ou privés. 


\title{
French Therapeutic Education Programme Aimed at Improving the Quality of Life of Laryngectomised Patients and their Close Relations: the Three Stages (Observational and Interventional Randomised) of the Study "PETAL"
}

Natacha Heutte, Moumainn Abouzayd, Laetitia Plisson, Mélanie Trocmé, Mélanie Seillier, Véronique Christophe, Lydia Guittet, Guillaume Grandazzi, Emmanuel “abin

\begin{abstract}
The therapeutic education of patients and their close relations is, as yet, poorly developed in France in the field of oncology. Total laryngectomy is a mutilating surgical procedure having a major impact on the patient's life, due to its physical and functional sequelae. Its psychosocial consequences are also important and alter the quality of life of patients and their close relations. Currently, care for laryngectomised patients consists essentially in informing and educating them on some technical procedures during hospital admission. The intervention of a speech therapist, often serves as the link between the patient and the hospital care team. These healthcare modalities often insufficiently account for the social, environmental and personal factors that interact in health-related problems. This report presents the therapeutic education programme protocol "PETAL" for laryngectomised patients and their close relations to improve their quality of life. The trial will be conducted over three phases: (1) the "pilot" phase aims at developing knowledge on the consequences of laryngectomy on the quality of life of patients and their close relations and developed a pluridisciplinary therapeutic education program, (2) the prospective intervention "replication" phase aims at evaluating the programme's transferability in three centres and (3) the cluster-randomised multicentric comparative intervention phase, will assess the benefits of the developed programme. Phase I identified nine themes of workshops related to therapeutic education, training and coordination of care. The developed programme should reinforce town-hospital links to improve help, follow-up and support for patients and their close relations.
\end{abstract}

Keywords: Therapeutic education · Quality of life · Total laryngectomy $\cdot$ Cancerology $\cdot$ Social psychology

\section{Introduction}

The therapeutic education of patients and their close relations is, as yet, poorly developed in France in the field of oncology [12], in particular for cancers of the upper aerodigestive tract [2]. In the case of pharyngeal and laryngeal cancer, total laryngectomy associated with radiotherapy remains a reference treatmentforadvanced-stage cancers. This mutilating surgical procedure has a major impact on the patient's life, due to its physical and functional sequelae: phonatory (loss of physiological voice), feeding, olfactory and aesthetic (tracheostomy). Its psychosocial consequences are also important, owing to the biographical disruption and the identity-related metamorphoses associated with illness and its treatment, which alter the quality of life not only of patients but also of their 
close relations. Indeed, transformation is not only at individual level, it also contaminates the life of close relations, in particular spouses, who share the day-to-day lives of patients [3]. Currently, careforlaryngectomised patients consists essentially in planning a specific appointment with a speech and language therapist before surgery and in informing and educating them on certain technical procedures (cannula replacement, mucosity aspiration, tracheostomy or phonatory implant cleaning) during hospital admission. Such education may be formalised and dispensed within the context of a therapeutic education programme. New voice education can be a long process and often involves the intervention of a speech therapist, who serves as the link between the patient and the hospital care team. These healthcare modalities often insufficiently account for the social, environmental and personal factors that interact in health-related problems. Despite the efforts engaged by healthcare teams within the framework of the French Cancer Plan aimed at improving care through a pluridisciplinary approach, important inadequacies remain, including insufficient town-hospital network and general practitioner implication, lack of support in solving psychosocial problems (professional rehabilitation, social isolation, identity reconstruction, etc.), absence of or insufficient attention afforded to close relations and the support they may personally need or offer the patient.

We are currently speculating on the possibility of improving the quality of life of laryngectomised patients and their close relations through the design, the implementation and the evaluation of a structured therapeutic education programme.

More specifically, the issue will be to determine what therapeutic education programme we should offer patients and their close relations in order to accompany them throughout their experience of laryngectomy and to reduce its impact on social and professional aspects of life.

The primary objective of "PETAL" is to design, implement and evaluate a patient therapeutic education (PTE) programme, for laryngectomised patients and their close relations, aimed at improving their quality of life. The secondary objectives of the study are as follows:

Phase 1: (1) To analyse the needs expressed by patients and their close relations, as well as healthcare professionals' expectations and current practice, in order to define the aims of the PTE programme (and consequently the programme's evaluation criteria); (2) to design, by a pluridisciplinary team, a programme that conforms to HAS (French National Authority for Health) recommendations, in particular through the inclusion of pluridisciplinary coordination modalities in the programme's structure, the reinforcement of town/hospital links and complementarity with the existing local PTE offer and (3) to implement the programme in order to verify its feasibility and to evaluate its quality.
Phase 2: (4) To deploy the programme in order to verify its transferability and to refine its qualitative evaluation. Phase 3: (5) To evaluate, within the framework of a randomised study, the programme's benefits for patients, their close relations and healthcare professionals, by developing the programme across a dozen healthcare centres in France and "elgium and (6) to evaluate the impact of patients' quality of life on close relations' quality of life 2 months, 6 months and 1 year after the patient's total laryngectomy.

\section{Material and Methods}

\section{Study Design}

The research will be conducted over three additional phases:

The first phase, referred to as the "pilot" phase, will include exploratory, observational and retrospective analysis aimed at developing knowledge on the consequences of laryngectomy on the quality of life of patients and their close relations, the strengths and weaknesses of current practice in patient support and the needs expressed by the players involved (patients, relations, professional carers). This analysis will be conducted via interviews with patients and their close relations and focus groups with both private and hospital sector healthcare professionals involved in patient care and support: head and neck surgeons, general practitioners, speech and language therapists, nurses and dieticians.

Our aim is to design, by a pluridisciplinary team, a therapeutic education programme for laryngectomised patients and their close relations. We will apply the quality assessment criteria recommended by the French National Authority for Health [7].

These quality criteria do not aim at initiating a current measurement involving references or standards of judgement, but at providing a number of questions for consideration by a workgroup in support of its mission to design a therapeutic education programme. We have chosen to select certain criteria as recommendations for the workgroup:

1. Include within the pluridisciplinary group different medical and paramedical professions, different sectors of activity (hospital, independent), different human science disciplines and representatives from patient associations, from the ethics committee and from administrative services

2. Design a programme suitable for pluridisciplinary implementation

3. Include within the programme the educative timescales required to cover the major events involved in laryngectomy and its post-operative evolution 
4. Provide for the programme to be adaptable to suit the needs of each patient, including alternating individual or group sessions

5. Evaluate the patient's subjective and objective needs (educative diagnosis) in order to identify learning priorities to develop a personalised path within the programme (active participation of each patient for the elaboration of his/her educational path)

6. Create an educative file to be integrated within the patient's medical file

7. Design a document that summarises the educative diagnosis in each patient's educative file

8. Create documents or procedures aimed at sharing information between different healthcare professionals

9. Include, for each educative session, an individual patient assessment (formative assessment)

10. Provide for the programme's coordination modalities (practice assessment, programme adaptation, readjustment based on evaluations, etc.)

11. Allow patients representatives to evaluate the programme and to improve it through the opportunity of forwarding feedback via the coordination group (process evaluation).

The programme will be tested in order to validate its feasibility and to make modifications that are coherent to the aforementioned quality criteria. This phase we refer to as the evaluation of the educative process by patients, their close relations and healthcare professionals. Over and above the aforementioned list, this evaluation may include the following:

1. Patient, close relation and healthcare professional satisfaction

2. The coherence between patient, close relation and healthcare professional expectations and the PTE programme's achieved aims

3. The skills developed by patients and their close relations

4. The features included in the PTE programme that facilitate the experience of laryngectomy by patients and their close relations

5. The features of the programme quoted by healthcare professionals as facilitating patient and close relation care and support, improving town/hospital links and reinforcing pluridisciplinary work

In 1998, the World Health Organisation [16] defined patient therapeutic education as aiming to "help patients and their families to understand the disease and the treatment, cooperate with health care providers, live healthily and maintain or improve their quality of life. [...] It takes into account [...] subjective and objective needs of patients, whether expressed or not." These needs were subsequently expressed in terms of skills requiring to be acquired by any patient suffering from chronic disease [4]:
1. Expressing his/her needs and determining goals in association with carers, informing close relations

2. Understanding, explaining oneself

3. Locating, analysing, measuring

4. Coping, deciding

5. Solving a day-to-day therapeutic problem involving managing his/her life and disease, solving a problem involving prevention

6. Practising, doing

7. Adapting, readjusting

8. Using the healthcare system's resources

9. Asserting his/her rights

Without foreseeing the results of phase 1 , we can nevertheless picture therapeutic education programme sessions for laryngectomised patients. For example, during initial education, the patient will need to acquire self-care skills (or rather techniques) such as relieving pain, loosening stiffness in the neck and shoulders, performing tracheal mucus aspirations, maintaining his/her cannula, readjusting his/her mastication and deglutition, managing breathing difficulties, performing the rehabilitation required to use his/her chosen means of communication, showering with due care, using a filter in order to reduce the introduction of dust or particles.

A distinction can be drawn between these self-care skills and those referred to as adaptation skills (or rather psychosocial or interpersonal skills), such as living temporarily with enteral feeding, choosing a substitution means of communication (oesophageal voice, tracheo-oesophageal voice, external electric prosthesis), minimising the impact of the operation on family, social and professional life.

Each of these skills can be targeted through the proposal of one or several individual and/or group sessions. For example, learning how to perform a technical act involved in cannula maintenance could be conducted at the patient's bedside over a few short individual sessions. In order to increase the patient's chances of acquiring such skills as comfortably as possible, the same skill "maintaining my cannula" could consequently also be the subject of a group session during which patients are invited to exchange views on their fears, on outside gaze, self-image or on other factors likely to be impacted by what is, at least in appearance, purely technical. Group sessions will offer an opportunity to welcome patients' emotions and to help them to exchange their points of view (or perhaps even to amend them), to facilitate the sharing of solutions or ideas between patients themselves and between patients and the care team. Close relation participation in these group sessions may also enable other aims to be targeted such as improved understanding of the patient and of his/her experience and difficulties. Hence, we can hope to see group sessions improve the conditions (both technical and emotional) in which specific acts are performed, whilst improving the 
opportunity for the close relation to offer more appropriate support to suit the patient's difficulties.

The HAS recommends the proposal of a comprehensive programme including regular follow-up sessions in order to reinforce acquired skills and knowledge, together with indepth follow-up sessions [7].

Regular follow-up could propose refresher training on the command of specific technical acts in order to limit potential infection, or a reminder on the best way to react in the case of signs of infection in order to avoid it worsening. Regular follow-up can also offer the opportunity to share with other patients a specific experience related to laryngectomy and likely to generate new difficulties requiring to be solved or strategies that patients have developed themselves to cope better with laryngectomy.

In-depth follow-up will involve events in the patient's life, changes in environment or projects that require the acquisition of new skills. One can imagine that a house move, a holiday or a job change oblige the patient to adapt, to develop solutions, to adopt new habits. Once more, the role of the close relation is essential both in terms of his/her participation and for developing personal skills aimed at helping the patient, or for personally coping better with a closely related patient.

In order to verify the potential for the programme's largescale deployment, the second phase, referred to as the prospective intervention "replication" phase, aims at evaluating the programme's transferability and quality in three centres.

The centres participating in this second phase already have experience in the development of PTE programmes and will be involved in the workgroup entrusted with the design of the PETAL (Programme d'Education Thérapeutique visant l'amélioration de la qualité de vie des Aidants et des patients opérés par Laryngectomie totale-Therapeutic education programme aimed at improving the quality of life of laryngectomised patients and their close relations, http:// www.laryngectomy.net/) programme during phase 1 .

During this phase, we plan to pursue the programme's qualitative evaluation and to define its feasibility criteria:

Necessary human resources (healthcare professional pluridisciplinarity, critical mass among professionals dedicated to therapeutic education, preparation time, education time, internal coordination time and educational team operation, time for exchange with external educational teams involved in the project and with other professionals involved in patient education)

Necessary material resources (supplies, rooms, educational material, educationfile, education notebook, correspondence with independent healthcare professionals)

Prerequisite resources (team training and experience in therapeutic education, active patient file, nearby partnership with players involved in patient education such as networks and patient associations)
The third phase, referred to as the "randomized" multicentric comparative intervention phase, should enable us to assess the benefits of the developed PTE programme on the quality of life of patients and their close relations.

We will validate the potential to implement the programme within these 12 centres involved:

- Number of centres having deployed the programme whilst adhering to its quality criteria

- Number of patients and close relations included in phase 3 of the study

- Patient and close relation participation rate in the PTE programme

- Patient, close relation, educational team healthcare professional and independent healthcare professional satisfaction rate

And, in particular, we will evaluate the programme in terms of patient and close relation quality of life scores, and number of skills developed by patients and their close relations.

The design of this evaluation phase is based on an original method: collective randomisation unit trials [5]. This type of trial is perfectly suited to studies aimed at evaluating intervention aimed at a higher level (carers) and when initiating two different care modalities do not appear feasible. This experimental set-up is widely used to evaluate the efficiency of patient education programmes [7].

In the present case, the aim is to compare the PETAL programme with existing practice in healthcare departments prior to the study's launch. In order to avoid centres randomised in the control group from finally refusing to participate in the study, precisely the same intervention will be scheduled within these centres, but at a 1-year interval (stepped wedge cluster randomised trials) [8, 11].

Participation in the present study may lead to modifications in patient care and support.

\section{Participants}

This study aims at improving the quality of life of laryngectomised patients and their close relations. We will apply the same inclusion criteria for the entire study (Table 1).

For the study's three phases, subjects participating in educational workshops will be patients having undergone laryngectomy and their close relations. These participants will have previously accepted to participate in the research project's given phase, and in the therapeutic education programme.

Throughout the study, we will call upon hospital and independent healthcare professionals and patient associations involved in laryngectomised patient support and having accepted to participate in the study (signed informed consent form).

The only exception to the above inclusion criteria involves phase A, during recruitment for semi-structured interviews 
Table 1 Inclusion and exclusion criteria for participation in the "PETAL" study

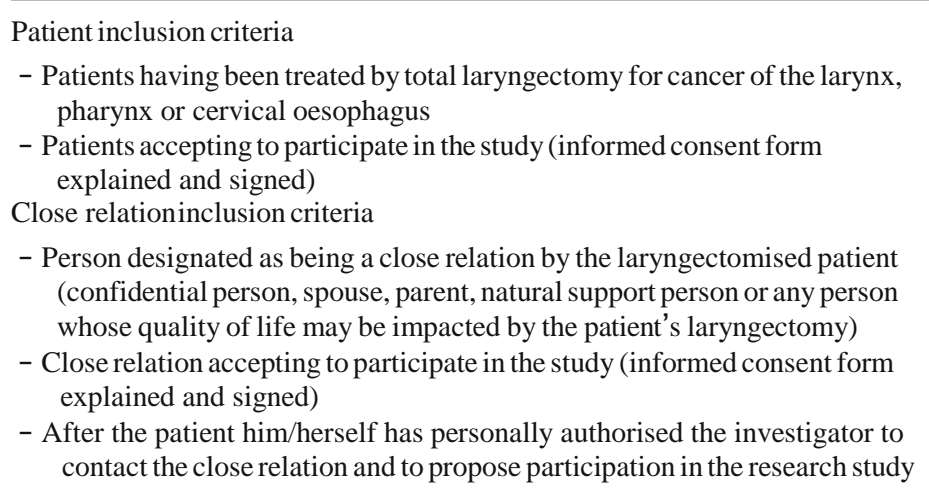

\author{
Patient exclusion criteria \\ - Physical, psychical, psychiatric or cognitive incapacity to respond \\ to questions or participate in interviews/sessions
}

Close relation exclusion criteria

- Physical, psychical, psychiatric or cognitive incapacity to respond to questions or participate in interviews/sessions aimed at analysing the impact of laryngectomy on the quality of life of patients and their close relations. Indeed, we hope to conduct interviews at various stages in the laryngectomy experience, i.e. at 1, 3, 6 and 12 months after surgery. We will consequently add the following inclusion criteria: time since laryngectomy.

\section{Judgement Criteria}

\section{Primary Endpoint}

The principal judgement criterion is the difference in gain observed at 6 months in the mean quality of life score of patients (PSS-HN scale-Performance Status Scale for Head and Neck cancer patients [10]) having benefited from the PTE programme and those having benefited from usual care during the third phase of the study.

\section{Secondary Endpoint}

Phase 1, Pilot Phase This exploratory, observational and retrospective phase aims at analysing the consequences of laryngectomy on patients and their close relations.

Via interviews with patients and close relations and focus groups involving healthcare professionals, together with an assessment of current healthcare, support and therapeutic education practice concerning laryngectomised patients, we will endeavour to identify the following:

- The impact of laryngectomy on the quality of life of patients and their close relations

- The expectations of patients and their close relatives in terms of educative intervention

- The expectations of healthcare professionals in terms of improved medical practice (patient and close relation support, and systems for exchanging information between the different players involved)
- Shortcomings in the current healthcare path for laryngectomised patients and their close relations

- "enefits in the current healthcare path with regard to patients' and their close relations' experience of laryngectomy

Phase 3 The secondary judgement criterion for phase 3 is the difference in gain observed at 6 months in the mean quality of life score (SF-36 scale-Short Form 36 health survey [14]) between close relations having benefited from the PTE programme and those having benefited from usual support.

\section{Statistical Methods}

\section{Sample Size Estimations}

The calculation of the number of subjects required is based on the comparison between mean total scores on the PSS-HN scale $[9,10] 6$ months after laryngectomy, taking into account cluster randomisation (patients from the same cluster are not independent), and putting forward the following hypotheses:

An anticipated improvement of 10 points in the mean score of patients in the intervention group (PETAL programme) compared to the control group (usual care) during the first period after implementation of the programme in the intervention group. Indeed, the PSS-HN scale has steps of 10 points and others of 25 points. Thus, the minimal improvement that could have a real clinical meaning seems to be a gain of 10 points.

Bilateral test and common variances in both groups (standard deviation of 20 points); an alpha risk of 5\%; a statistical power of $80 \%$; an intraclass correlation coefficient of 0.05 ; a total of 6 clusters per randomisation arm.

The estimated number of required subjects is as follows:

Phase 1, exploratory, observational and retrospective: 40 patient/close relation couples 
Phase 2, prospective, interventional: 30 patient/close relation couples

Phase 3, comparative, interventional, prospective, multicentric: 264 patient/close relation couples

\section{Statistical Analyses}

Statistical analysis will be based on intention to treat. Patient characteristics are described according to the two arms, and according to the cluster, they are included in Fig. 1. We will initially compare patients' total PSS-HN scores at 6 months between both arms, taking into account data correlation (generalised estimating equation model).

We will conduct global analysis taking into account the study's different periods, clusters and the intervention scheduled for each period, using a generalised linear mixed model for the total scoreandforallsub-scales [8]. This analysis willbe adjusted according to patients' demographic and medical characteristics.

Similarly, we will conduct global analysis on close relations' quality of life for all SF-36 sub-scales. This analysis will be adjusted for close relation demographic characteristics and for patient medical and quality of life characteristics.

\section{Study Progression}

\section{Recruitment}

All patients and their close relations who satisfy all of the selection criteria are likely to be included in one of the three study phases, depending on the place and date of their hospitalisation. Independently of the study phase, the investigator will provide them with the explanatory letter and will request that the patient and close relation sign the document, hence confirming their informed consent for the study phase in which they agree to participate. An identification number will then be assigned to each participant according to a chronological inclusion order at the investigation centre. The eligibility form, on which the person's identification number will be noted, will then be forwarded to the study promoter in order to register the inclusion.

A similar procedure will be used to inform and to verify the informed consent of healthcare professionals accepting to participate in the study.

\section{Phase 1: Pilot Phase (Exploratory, Observational, and Retrospective)}

An individual semi-structured interview integrating sociological investigation methods will be proposed to patients and to close relations accepting to participate in this first phase. Patients and their close relations will be heard at different times during their experience of laryngectomy. We will question patients and close relations, 1, 3, 6 and 12 months after total laryngectomy, on the consequences of the operation on their quality of life, their expectations and their needs in terms of therapeutic education and support, as well as on the features of available care/intervention that help them to cope with their situation. Healthcare professionals will be called upon to participate in focus groups on the features to be maintained, improved or created in order to optimise the care and support offered to laryngectomised patients and their close relations in an aim to limit the impact of treatment on their quality of life. Professionals will be interviewed only once; however, this interview will cover patient and close relation care and support at different stages of the healthcare path.

For phase 1 , we will question 10 patient/close relation couples at each of these different intervals after total laryngectomy, i.e., a total of 40 couples. This qualitative evaluation will
Fig. 1 The "randomized" multicentric comparative intervention phase 3 study design

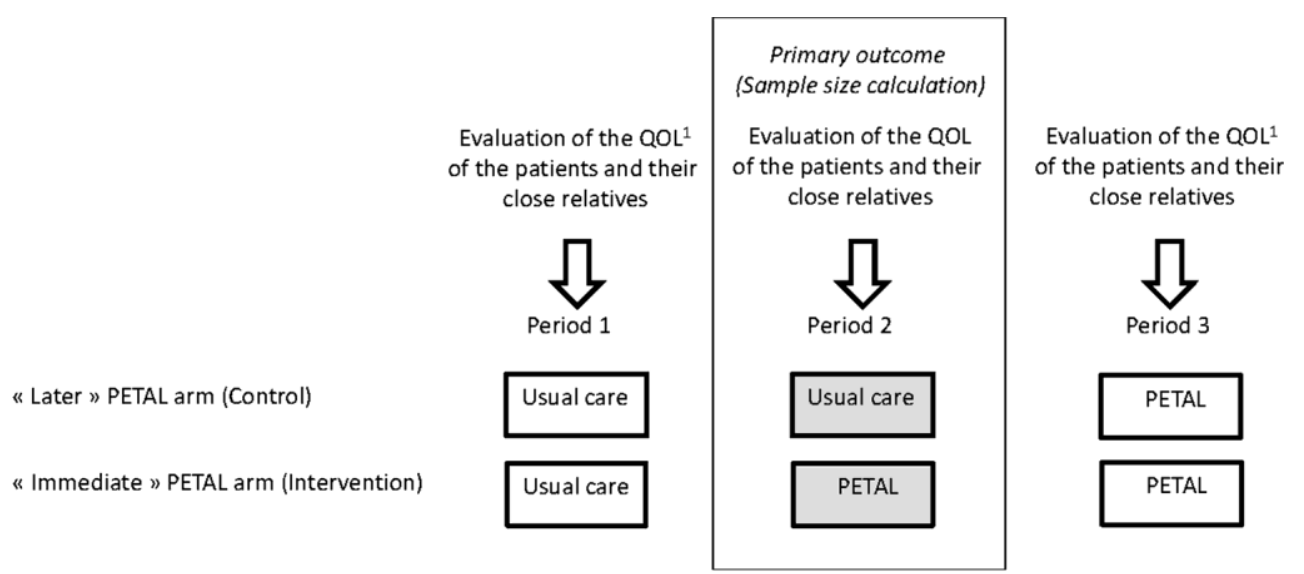

Each arm is composed of 6 clusters, all patients of the same cluster receive the same care (PETAL ou usual care) at the same period.

${ }^{1}$ QOL: Quality Of Life 
be conducted in the four centres in Caen and Lille (CHU: Centre Hospitalier Universitaire-University Hospital, and CLCC: Centre de Lutte Contre le Cancer-Cancer Centre). Concurrently, five focus groups will be formed in Caen and will include public and independent healthcare professionals involved in laryngectomised patient care.

Analysis of interviews and focus groups will serve as the basis for the project's participating teams to elaborate, in a pluridisciplinary manner, a structured therapeutic education programme for laryngectomised patients and their close relations. This design phase will also offer the opportunity to identify which skills are to be developed by patients and their close relations, hence defining the education programme's evaluation criteria.

This first phase will be completed with a test of the programme's feasibility, conducted within the study's coordinating physician's practicing centre. A group of patients will consequently be recruited for participation in the programme's feasibility study. Details on how the programme will unfold will be included in the explanatory letter enclosed with the informed consent form.

Although we cannot detail the programme in the present document, we do know that the educative approach will include the following:

- One or several individual interviews in order to reach an educative diagnosis

- The definition of a personalised programme with and for the patient

- The implementation of individual, group or alternating sessions

- Time for assessing the patient's acquired skills (i.e. the programme's aims)

Debriefing time with patients and their close relations after each educative period (from the educative diagnosis to evaluation) will enable the education programme's coordination group to make improvements based on participants' comments

During this first phase, in complement to a nurse previously trained in PTE, a second nurse and a speech therapist from the coordinating centre will be trained by the CERFEP (CEntre de Ressources et de Formation à l'Education du patient-Centre of resources and training in patient education).

\section{Phase 2: Replication (Prospective, Interventional)}

Following agreement and informed consent to participate in phase 2 of the study, as described above, patients and their close relations will be invited to benefit from the therapeutic education programme developed following phase 1 .

Phase 2 will be conducted in three centres.
Healthcare professionals having accepted to participate in phase 2 of the study will be questioned on the features that facilitate healthcare, improve town/hospital links and reinforce pluridisciplinary work.

Once this test phase completed, the coordination group will verify its adequacy with the aforementioned quality criteria, then will determine the feasibility indicators to be used to deploy the programme throughout other centres.

Phase 3: Randomisation (Comparative, Interventional, Prospective, Multicentric)

Centres participating in this third phase have no existing PTE trained staff. Inclusion of these centres in the randomised trial consequently implies dispensing PTE training to two carers per centre. This training will be dispensed by the CERFEP and funded within the framework of the project, hence guaranteeing equivalent training dispensed in all centres. In order to ensure that all patients from all centres benefit from the PETAL programme, it will be implemented both in the intervention group and the control group, but at a 1-year interval.

This third study will involve deploying the programme throughout 12 centres, hence enabling the quantitative evaluation of the programme's effects and the generalisation, among several sites, of its feasibility criteria (i.e. its transferability).

Assessment For phase 3 (randomised study), assessment of the quality of life of patients will rely on the use of several scales, all of which have validated French versions: PSS-HN, QLQ-C30 (Quality of Life Questionnaire Cancer [1]) and QLQ-HN35 (Quality of life Questionnaire Head and neck(13)) from EORTC (European Organisation for research and treatment of cancer), VHI (Voice Handicap Index [13, 15]) and GHQ-12 (General Health Questionnaire [6]). Quality of life of close relations will be assessed using the French version of the SF-36 scale.

These assessments will be conducted 2 months, 6 months and 1 year after the patient's total laryngectomy.

\section{Ethics}

The study will include patients and close relations accepting to participate in the study (informed consent form explained and signed). The study has obtained all the relevant authorisations (no. A12-D47-VOL.14-PETAL) for the protection of patients enrolled in clinical trials (comité de protection des personnes CPP).

\section{Preliminary Results}

In a first step, we interviewed 41 patients and caregivers. This work allowed us to identify the main life features of the people interviewed and to classify it in three groups: therapeutic 
education, cure and formation. Nine items have been selected to divide therapeutic education features:

\section{Communication}

2. Own image and look of others

3. sequellae and complications of treatments

4. Rule of caregivers

5. "ereavement

6. Closeness

7. Means

8. Coping and emotivity

9. Treatment cure

You can find workshops on website: laryngectomy.net

\section{Conclusion}

This is a pluridisciplinary study via which we aim to improve the quality of life of laryngectomised patients and their close relations through the design and the sustainable deployment of an innovative PTE programme in France and Belgium. Furthermore, we hope that this programme will have as a side-benefit to strengthen the city-hospital network and the communication between health professionals, in order to indirectly improve the care of patients and their close relations.

Funding This project was made possible by funding from the Institut National du Cancer (INCA), within the framework of 2012 No. 12-220.

\section{References}

1. Aaronson NK, Ahmedzai S, Bergman B, Bullinger M, Cull A, Duez NJ, Filiberti A, Flechtner H, Fleishman SB, de Haes JC (1993) The European Organization for Research and Treatment of Cancer QLQ-C30: a quality-of-life instrument for use in international clinical trials in oncology. J Natl Cancer Inst $85: 365-376$
2. Allison P, Edgar L (2010) Le programmeNucare chez les personnes atteintes d'un cancer des voies aérodigestives supérieures. In Foucaud J, Bury JA, Balcou-Debussche M, Eymard C (dir.) Education thérapeutique du patient. Modèles, pratiques et évaluation, Saint-Denis, INPES, pp 271-87

3. Babin E, Grandazzi G (2010) Vivre avec le cancer. La vie des conjoints de patients laryngectomisés. Psycho-Oncology 4(4): 303-308

4. D'Ivernois JF, Gagnayre R (2001) Mettre en oeuvre l'éducation thérapeutique. ADSP 36:11-13

5. Donner A, Klar N (2000) Design and analysis of cluster randomization trials in health care. Oxford University Press, New York

6. Hankins M (2008) The reliability ofthe twelve-item general health questionnaire (GHQ-12) under realistic assumptions. BMC Public Health 8:355

7. Haute Autorité de Santé (HAS) (2007) Structuration d'un programme d'éducation thérapeutique du patient dans le champ des maladies chroniques. Guide méthodologique

8. Hussey MA, Hughes JP (2007) Design and analysis of stepped wedge cluster randomized trials. Contemp Clin Trials 28:182191

9. List MA, D'Antonio LL, Cella DF, Siston A, Mumby P, Haraf D, Vokes E (1996) The performance status scale for head and neck cancer patients and the functional assessment of cancer therapyhead and neck scale. A study of utility and validity. Cancer 77: 2294-2301

10. List MA, Ritter-Sterr C, Lansky SB (1990) A performance status scale for head and neck cancer patients. Cancer 66:564569

11. Mdege ND, Man M-S, Taylor Nee BrownCA, TorgersonDJ (2011) Systematic review of stepped wedge cluster randomized trials shows that design is particularly used to evaluate interventions during routine implementation. J Clin Epidemiol 64:936-948

12. Pérol D, Toutenu P, Lefranc A, Régnier V, Chvetzoff G, Saltel P, Chauvin F (2007) Therapeutic education in oncology: involving patient in the management of cancer. Bull Cancer (Paris) 94:267-274

13. Rosen CA, Lee AS, Osborne J, Zullo T, Murry T (2004) Development and validation of the voice handicap index-10. Laryngoscope 114:1549-1556

14. Ware JE, Sherbourne CD (1992) The MOS 36-item short-form health survey (SF-36). I. Conceptual framework and item selection. Med Care 30:473-483

15. Woisard V, Bodin S, Puech M (2004) The voice handicap index: impact ofthe translation in French on the validation. Rev Laryngol Otol Rhinol 125:307-312

16. World Health Organisation BR pour l'Europe (1998) Éducation thérapeutique du patient, programme de formation continue par des professionnels de soins dans le domaine de la prévention des maladies chroniques. Recommandations d'un groupe de travail de l'OMS, Copenhague. Version française UCL Bruxelles 